\title{
Transverse Spin Relaxation Rate
}

National Cancer Institute

\section{Source}

National Cancer Institute. Transverse Spin Relaxation Rate. NCI Thesaurus. Code C80362.

The relaxation rate of spin polarization in the plane perpendicular to the magnetic field of a spectrometer back to zero as precessing spins loose coherence with each other. 